Article

\title{
Biomimetic Properties of Force-Spun PHBV Membranes Functionalised with Collagen as Substrates for Biomedical Application
}

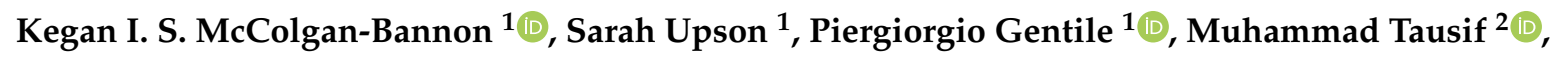 \\ Stephen Russell ${ }^{2}$, Kenneth Dalgarno ${ }^{1}$ and Ana Marina Ferreira ${ }^{1, *}$ (i) \\ 1 School of Engineering, Newcastle University, Newcastle-Upon-Tyne NE1 7RU, UK; \\ K.Mccolgan-Bannon2@newcastle.ac.uk (K.I.S.M.-B.); sarahjane936@outlook.com (S.U.); \\ piergiorgio.gentile@ncl.ac.uk (P.G.); kenny.dalgarno@newcastle.ac.uk (K.D.) \\ 2 Nonwovens Research, School of Design, University of Leeds, Leeds LS2 9JT, UK; m.tausif@leeds.ac.uk (M.T.); \\ S.J.Russell@leeds.ac.uk (S.R.) \\ * Correspondence: ana.ferreira-duarte@ncl.ac.uk; Tel.: +44-(0)191-208-7679
}

Received: 3 May 2019; Accepted: 24 May 2019; Published: 28 May 2019

\begin{abstract}
The force-spinning process parameters (i.e., spin speed, spinneret-collector distance, and polymer concentration), optimised and characterised in previous work by this group, allowed the rapid fabrication of large quantities of high surface area poly(3-hydroxybutyric acid-co-3-hydroxyvaleric acid) (PHBV) polymeric fibre membranes. This paper examined the potential application for force-spun PHBV fibres functionalised with type I collagen for tissue regeneration applications. PHBV fibre scaffolds provide a biologically suitable substrate to guide the regeneration of dermal tissues, however, have poor cellular adhesion properties. The grafting of collagen type-I to PHBV fibres demonstrated improved cell adhesion and growth in Neo-NHDF (neonatal human dermal fibroblasts) fibroblasts. The examination of fibre morphology, thermal properties, collagen content, and degradability was used to contrast the physicochemical properties of the PHBV and PHBV-Collagen fibres. Biodegradation models using phosphate buffered saline determined there was no appreciable change in mass over the course of 6 weeks; a Sirius Red assay was performed on degraded samples, showing no change in the quantity of collagen. Cell metabolism studies showed an increase in cell metabolism on conjugated samples after three and 7 days. In addition, in vitro cytocompatibility studies demonstrated superior cell activity and adhesion on conjugated samples over 7 days.
\end{abstract}

Keywords: PHBV; collagen; force spinning; functionalization; tissue engineering

\section{Introduction}

Polyhydroxylalkanoates (PHAs) are a class of biocompatible, biodegradable polymers produced by microorganisms and through plant-based fermentation [1]. Of this class, one of the most well-studied variants is poly(3-hydroxybutyric-co-3-hydroxyvaleric acid) (PHBV), a copolymer of polyhydroxybutyrate and polyhydroxyvalerate. PHBV is a biodegradable, non-antigenic, and biocompatible type of microbial polyester, where low molecular weight PHB (polyhydroxybutyrate) has been found in cells. Due to its favourable biological properties, PHBV has been targeted for applications in a range of tissue engineering (TE) and in vivo applications, including wound dressings, neural, dermal, osseous and cardiovascular tissues [2-5].

Nanofibres have been shown to be of value for TE by mimicking the fibrillary structure of the extracellular matrix (ECM) [6]. Electrospinning is an established technique for the preparation of nanofibres with diameters ranging from tens of nanometres to several microns [4]. An ideal scaffold 
for TE should have an interconnected porous structure [7]; as porous microfiber scaffolds allow for the full integration of cells throughout the scaffold matrix.

Forcespinning has presented itself as a viable, low-cost alternative to electrospinning. Forcespinning functions by ejecting polymer solution from spinnerets at high rotational speeds onto collecting frames, enabling the production of fibres from a wide range of materials and at higher rates than the electrospinning technique [8]. Moreover, as forcespinning uses centrifugal forces rather than electrical forces as in the electrospinning technique, processing fibres by forcespinning does not require a high voltage electric field or a conductive polymer solution [8]. As many materials can be forcespun into fibres with this method, this versatile method is a promising alternative to quickly produce the extracellular matrix (ECM) mimicking fibre membranes from biopolymers of interest for tissue engineering. The authors have reported in previous work [9], forcespun PHBV membranes with fibre diameters between 0.5 and $3 \mu \mathrm{m}$, using chloroform as a solvent. A PHBV polymer concentration of $25 \% w / v$ spun at $9000 \mathrm{r} / \mathrm{min}$ produced the highest quality fibres, and with a spinneret to collector distance of $39.2 \mathrm{~cm}$, producing the equivalent of $11 \mathrm{~km}$ of fibre per minute, per needle. The rapid rate of production, mechanical properties (3 MPa average tensile strength), and biocompatibility of PHBV fibres, opens an extensive potential range of biomedical applications.

As a means to tailor the biological or mechanical function of PHBV scaffolds, blends, and composites with other natural or synthetic biopolymers [10-14], and bioceramics [15-18] have been studied. In particular, collagen type-I, which is the most abundant protein in the ECM [19] and responsible for the integrity of the tissue, is one of the most commonly investigated materials for TE scaffolds and as a coating or component in composite TE scaffolds [20-22]. Collagen type-I possesses structural motifs to which cell integrins bind [23], mediating cellular adhesion and migration in the native ECM [24]. To imbue scaffolds with these properties, collagen type-I is commonly combined with synthetic biocompatible polymers like poly- $\varepsilon$-caprolactone and poly-lactic acid, to improve cell growth and adhesion. Likewise blends of PHBV and collagen have been reported, with the incorporation of collagen resulting, in all cases, in superior cellular behaviour over pure PHBV scaffolds. Han et al. [2] examined the co-culturing of hair follicular epithelial cells with dermal sheath cells on electrospun scaffolds of PHBV, PHBV/Collagen, and PHBV/gelatin. PHBV/collagen blends not only showed the greatest extent of cell growth, but also the expression of extracellular materials, i.e., type I collagen, smooth muscle actin, and elastin. While a study by Prabhakaran [18] with similarly prepared electrospun scaffolds, compared PHBV and PHBV/collagen composites, in random and aligned orientations, as substrates for nerve regrowth. Composite scaffolds with a higher ratio of collagen content displayed higher levels of cell growth, and cells were observed to align with the orientation of composite fibres. However, no differences in the total cell proliferation were observed between different fibre orientations, indicating that the driver in cell growth was collagen content.

PHBV is known to be a hydrophobic polyester without many functional groups available for a covalent biofunctionalisation [25]. In this regards, different efforts have been made for incorporating biological cues in order to render this material more attractive for cells and favour integration with surrounding tissues. Biazar et al. [26], fabricated nanofibre PHBV scaffolds using microwave plasma, afterwards submerged in a collagen solution at $50^{\circ} \mathrm{C}$, a method that may damage the side chain motifs to which cells bind. In a study of the use of somatic stem cells on PHBV-collagen electrospun membranes for skin defects [5], Keshel et al. immobilised collagen via the plasma method onto nanofibrous PHBV scaffolds. Their approach found that the graft of collagen-coated nanofibrous PHBV scaffolds loaded with USSC cells increased cellular growth and improved the healing process of skin defects in rat models. In the studies where the PHBV and collagen were electrospun concurrently in fluorinated solvents (e.g., hexafluoroisopropanol or trifluoroethanol), there was no conclusive characterisation of the collagen. This leads to contention as an article [27] examining the electrospinning of collagen nanofibres showed the denaturing effect of highly teratogenic [28] fluorinated solvents on collagen during the electrospinning process. This study and the lack of collagen characterisation in membranes spun from fluorinated solvents indicates benefit in the post-spinning conjugation of collagen to 
spun fibre scaffolds to preserve the triple helical structure. However, in the case of Han et al. [2], concurrently spun PHBV/collagen in fluorinated solvents still demonstrate superior cellular growth than PHBV/gelatin scaffolds prepared in the same manner.

In this work, forcespun PHBV fibres optimised in previous work by this group [9], have been grafted with type I collagen using the easy, efficient, and low-toxicity carbodiimide coupling chemistry. The grafting of collagen molecules aims to incorporate biofunctional motifs onto forcespun PHBV nanofibres, enhancing the biological properties of the fibre scaffold and improving the adhesion while preventing collagen loss in vitro through the use of covalent crosslinking to the polymer scaffolds. In this paper, we propose a new approach that combines forcespinning-fabricated membranes and the simple and efficient carbodiimide chemistry as a production method for fabricating in a quicker and scalable manner, collagen-functionalised nano/microfiber membranes, in the absence of toxic processing solvents that can lead to undesirable effects. This approach of fabricating PHBV-Collagen scaffolds and the physical-chemical properties of functionalised fibres were evaluated, along with cytocompatibility using human fibroblast cells, in order to demonstrate the suitability for tissue engineering and regenerative medicine applications.

\section{Materials and Methods}

\subsection{Materials}

Poly(3-hydroxybutyric-co-3-hydroxyvaleric acid) (PHBV) (12\% valerate, Sigma Aldrich, St. Louis, MO, USA), Chloroform (99.8\% reagent grade, Sigma Aldrich), N-(3-Dimethylaminopropyl)$N^{\prime}$-ethylcarbodiimide hydrochloride (EDC) (Sigma Aldrich), N-Hydroxysuccinimide (NHS), 2-(N-Morpholino) ethanesulfonic acid hydrate (MES) (Sigma Aldrich), Sodium Hydroxide (Sigma Aldrich), Tween 20 (Sigma Aldrich), Dulbecco's Phosphate Buffered Saline (PBS) (without calcium chloride, liquid, sterile-filtered, Sigma Aldrich), DAPI (Sigma Aldrich), Dulbecco's Modified Eagle Medium (DMEM) (high glucose, Thermo Scientific, Waltham, MA, USA), Live/Dead cytotoxicity kit (Thermo Scientific), Collagen type I Pepsin-extracted $6 \mathrm{mg} / \mathrm{mL}$ (Collagen Solutions Plc., London, UK), were purchased and used without further purification. Sirius Red collagen staining kit was purchased from Biocolor (Antrim, UK). The deionized water used in the experiments was obtained using a Purite Suez Edi-60 with Suez PP8 and PT8 (Paris, France) purification cartridges equipped.

\subsection{Methods}

\subsubsection{Preparation of PHBV Fibre Membranes and Films}

A solution of $25 \%$ w/v PHBV in chloroform was prepared by stirring for $12 \mathrm{~h}$ in ambient conditions. A FibeRio 1000M Forcespinning ${ }^{\mathrm{TM}}$ machine (McAllen, TX, USA) was used to produce the samples. For each spin cycle, $1.6 \mathrm{~mL}$ of solution was injected into a hollow fluid reservoir, which was tipped with two hypodermic needles (30 gauge with a shaft length of $12.5 \mathrm{~mm}$ ). The collector was located radial to the spinneret and comprised a series of metallic bars set at $39.2 \mathrm{~cm}(\mathrm{OD})$ from the spinneret at $9000 \mathrm{rpm}$ (as reported in Figure 1). Cardboard rectangular frames of dimensions 180 and $85 \mathrm{~mm}$ were affixed to the bars to facilitate the collection of flat areas of the fibrous web.

Films of PHBV were prepared by solvent casting. PHBV pellets, $5 \% w / v$, in chloroform were added to $100 \mathrm{~mL}$ of chloroform and stirred until fully dissolved. The solution was cast into a $15 \mathrm{~cm} \varnothing$ diameter petri dish and the solvent removed overnight under gentle air flow. 

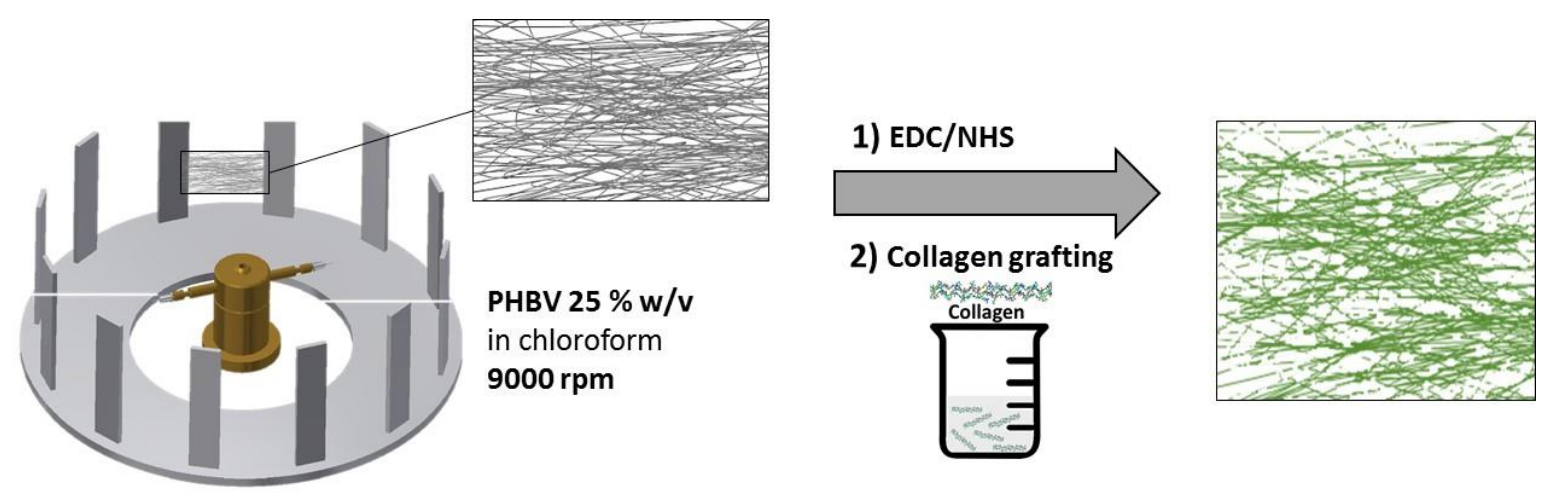

Figure 1. Schematic representation of the fabrication process for the PHBV-Collagen enhanced scaffolds using forcespinning and carbodiimide coupling.

\subsubsection{Collagen Immobilisation}

Collagen type I was immobilised onto the PHBV fibre membranes using the following protocol. PHBV fibrous mats were immersed into $1 \mathrm{M}$ sodium hydroxide solution at room temperature for $3 \mathrm{~min}$. Then, the $\mathrm{NaOH}$ solution was removed and the samples were washed with distilled water. Alkali-treated fibrous mats were submerged into a solution containing $10 \mathrm{mM}$ of EDC and $10 \mathrm{mM}$ of NHS in MES buffer $(\mathrm{pH}=6)$ for $6 \mathrm{~h}$. Activated PHBV mats were submerged into a collagen solution $(1 \mathrm{mg} / \mathrm{mL}$ in acetic acid solution, $\mathrm{pH} 6)$ overnight at $4{ }^{\circ} \mathrm{C}$. The obtained samples were washed abundantly with distilled water.

\subsubsection{Fibre Morphology}

The surface morphology of forcespun PHBV fibres was analysed using environmental scanning electron microscopy (ESEM, TESCAN VEGA LMU, Newcastle University, Newcastle, UK) at an accelerating voltage of $15 \mathrm{kV}$ at $600 \times$ and $800 \times$ magnifications.

\subsubsection{ATR-FTIR (Attenuated Total Reflection, Fourier-Transform Infrared Spectroscopy)}

The PHBV films and fibrous mats before and after collagen grafting were examined by mid-infrared spectroscopy. FTIR spectra of 36 scans at $4 \mathrm{~cm}^{-1}$ resolution, within the range of $4000-600 \mathrm{~cm}^{-1}$, were obtained by a Frontier PerkinElmer FT-IR spectrophotometer (PerkinElmer, Waltham, MA, USA) with a universal attenuated total reflectance (ATR) module in the diamond.

\subsubsection{XPS}

The surface composition was determined by X-ray photoelectron spectroscopy (XPS; Theta Probe, Thermo Scientific, East Grinstead, UK), which uses a micro-focused AlKa X-ray source (1486.6 eV), operated with a $400 \mu \mathrm{m}$ spot size (100 W power). Survey spectra were collected at pass energy of $200 \mathrm{eV}$, a step size of $1 \mathrm{eV}$, and a dwell time of $50 \mathrm{~ms}$, with the spectrometer operated in standard (not angle-resolved) lens mode. High-resolution regional spectra were collected using pass energy of $40 \mathrm{eV}$, a step size of $0.1 \mathrm{eV}$, and a dwell time of $200 \mathrm{~ms}$. High-resolution spectra envelopes were obtained by curve fitting the synthetic peak components using the software CasaXPS (Version 2.3.19).

\subsubsection{Collagen Quantification-Sirius Red Assay}

The amount of collagen present in the coated PHBV fibres and films was quantitatively determined using the Sirius Red dye kit. Films and fibrous mats were cut into square samples with a surface of $1 \mathrm{~cm}^{2}$ and then incubated in $1 \mathrm{~mL}$ of Sirius Red dye in a cap tube for $30 \mathrm{~min}$, forming a collagen-dye complex following the protocol provided by Sircol. Thereafter, the dye solution was drained, and the stained samples were washed twice with cold washing solution, removing the unbound dye. The washing solution was removed along with any fluid from the lip of the Eppendorf tubes using 
cotton wool buds. The bound dye was dissolved using $250 \mu \mathrm{L}$ of the provided alkali solution to the samples and mixed for $10 \mathrm{~min}$. Then, $200 \mu \mathrm{L}$ of collagen bound-dye solution was transferred into a 96-well plate, and optical density was measured at $555 \mathrm{~nm}$ with UV-Vis scanning spectrophotometer (Sunrise, TECAN, Mendendorf, Switzerland) using XFluor4 software (TECAN, V4.51). The quantity of collagen bonded to PHBV films and fibres was calculated using a calibration curve prepared with known collagen concentrations. All the measurements were performed in triplicate.

\subsubsection{Contact Angle}

The fibre membrane surface static contact angles were investigated at room temperature using a Contact Angle CAM 200 KSV Instrument (KSV Instruments, Helsinki, Finland), equipped with One Attension Theta software using the sessile drop method, dispensing $5 \mu \mathrm{L}$ water droplets (HPLC grade) directly onto the sample surface. Contact angle measurements were analysed using automated software and presented as the average of 10 measurements from three different points on each surface.

\subsubsection{Thermal Analysis}

Differential scanning calorimetry (DSC) was used to investigate changes in the thermal properties of PHBV fibre membranes before and after collagen conjugation. Samples (4-8 mg, aluminium pans) were analysed using a Netzsch Polyma, Netzsch Proteus software. A heating scan from 20 to $200^{\circ} \mathrm{C}$ was conducted at a rate of $10{ }^{\circ} \mathrm{C} \mathrm{min}-1$ under a nitrogen atmosphere.

\subsubsection{Hydrolytic Degradation}

In vitro biodegradation studies were performed in Phosphate Buffered Saline (PBS) solution, $\mathrm{pH}$ 7.4 , at $37^{\circ} \mathrm{C}$ (VWR, Incu-Line). Differences in mass were measured by subtracting the dry weight after incubation at time points from the initial dry weight. The material was removed from incubation, washed with deionised water, and dried in a vacuum desiccator before weighing.

\subsubsection{Cell Culture}

Neo-NHDF fibroblasts were grown at $37{ }^{\circ} \mathrm{C}, 5 \% \mathrm{CO}_{2}$, in Dulbecco's Modified Eagle Medium (DMEM, Sigma Aldrich, UK), $4.5 \mathrm{~g} / \mathrm{L}$ glucose, supplemented with 10\% foetal bovine serum (FBS) and a $1 \%$ antibiotic mix containing penicillin and streptomycin $(100 \mu \mathrm{g} / \mathrm{mL})$. Circular force-spun samples of $10 \mathrm{~mm}$ diameter (thickness of $127 \pm 5.8 \mu \mathrm{m}$ ) were secured in cell well inserts (Cellcrown 24, Scaffdex, Tampere, Finland), positioned in 12 well plates (Tissue culture treated, 3526, Costar), and UV-sterilized at $254 \mathrm{~nm}$ for $30 \mathrm{~min}$. DMEM was placed into each well up to the level of the scaffold surface, pre-wetting the material for $30 \mathrm{~min}$ before cell seeding. A suspension of 40,000 cells in DMEM was seeded onto the surface of each scaffold (incubated at $37^{\circ} \mathrm{C}, 5 \% \mathrm{CO}_{2}$, for $30 \mathrm{~min}$, then the wells were topped up with DMEM to a volume of $1.5 \mathrm{~mL}$.

\subsubsection{Cytotoxicity (Live/Dead)}

Cell viability was assessed with live/dead staining (LIVE/DEAD ${ }^{\circledR}$ Imaging Kit, Life Technologies, Thermo Fisher Scientific) at days one and three. Samples were washed with PBS and stained with $1.5 \mathrm{~mL}$ solution of $1 \mu \mathrm{L}$ calcein AM and $2 \mu \mathrm{L}$ Ethidium homodimer- 1 per mL in PBS. After $30 \mathrm{~min}$ of incubation at room temperature, the scaffolds were mounted on glass slides and an area of $3.6 \mathrm{~mm}^{2}$ was imaged with DLMB-Lecia microscope, using Spot Advanced software.

\subsubsection{Metabolic Activity}

Metabolic activity was assessed by Presto Blue assay (Life Technologies, Thermo Fisher Scientific). Cell culture media was removed from samples and replaced with $1500 \mu \mathrm{L}$ of 1:9 v/v Presto Blue to DMEM. Treated samples were incubated at $37{ }^{\circ} \mathrm{C}, 5 \% \mathrm{CO}_{2}$, for $2 \mathrm{~h}$ after which the solution was 
removed and $200 \mu \mathrm{L}$ from each sample placed into an opaque 96-well plate. Fluorescence readings were measured using a Gemini XPS and EM Microplate Reader (Molecular Devices, Wokingham, UK)

\subsubsection{Staining Cellular Cytoskeleton (Confocal)}

Cell morphology was observed using cytoskeleton and nucleus stains. After culture, samples were fixed using $4 \%$ paraformaldehyde for $15 \mathrm{~min}$ at room temperature, after which samples were washed with PBS/tween (0.1\%) three times. $1500 \mu \mathrm{L}$ of Rhodamine-Phalloidin solution (prepared to 1:1000 dilution in PBS) was added to each sample for $30 \mathrm{~min}$ at room temperature, after which the solution was removed, and samples were washed thrice with deionised water. DAPI (1 mg/mL in PBS) was added to each well for $30 \mathrm{~min}$ at room temperature; samples were then washed with PBS before mounting on microscope slides. Images were obtained with a Nikon AR1 inverted microscope using Nikon NIS elements software.

\subsection{Statistical Analysis}

Analytical tests were performed in triplicate to attain statistical validity. Statistical analysis was determined using Prism 7 software (GraphPad). The threshold for significance was determined at $p<0.05$.

\section{Results}

\subsection{Scaffold Characterisation}

\subsubsection{Fibre Collagen Content and Morphology}

Figure 2 depicts fibre morphologies of PHBV membranes before and after collagen conjugation. Fibre diameter in unconjugated samples ranged between 731 and $5.40 \mu \mathrm{m}$ (median $1.67 \mu \mathrm{m}$, $\mathrm{SD} \pm 1.27 \mu \mathrm{m}$ ), and the range observed in conjugated fibres was between 631 and $6.85 \mu \mathrm{m}$ (median $1.64 \mu \mathrm{m}, \mathrm{SD} \pm 1.99 \mu \mathrm{m}$ ). Collagen content was measured as a function of surface area and sample mass. Conjugated casted films were observed to contain on $8.3 \pm 1.1 \mu \mathrm{g} / \mathrm{cm}^{2}$ of collagen per area of film, while conjugated nanofibres detected values of $25.4 \pm 3.1 \mu \mathrm{g} / \mathrm{mg}$ of collagen per mass of polymeric mesh.

A
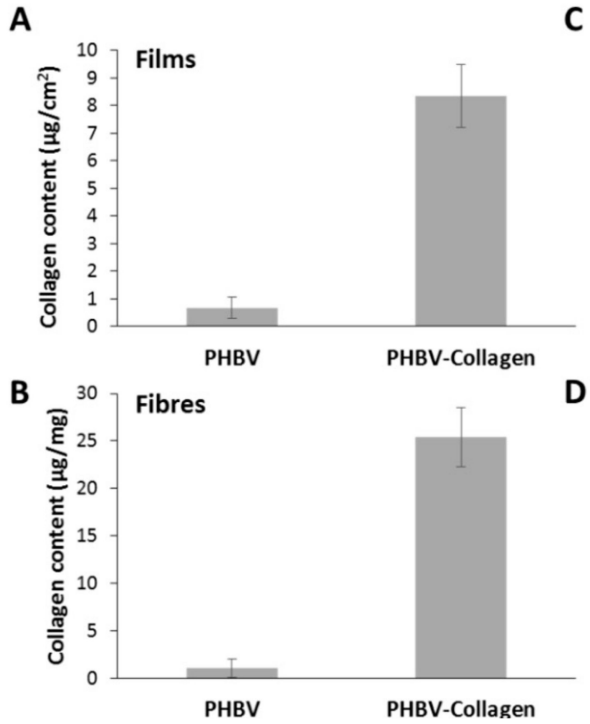

C
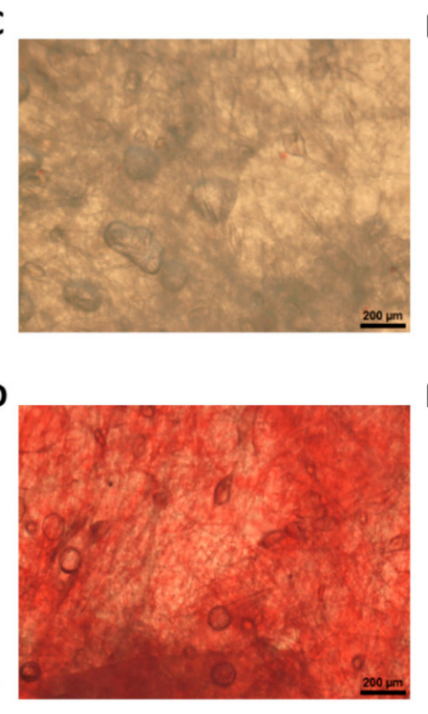

E

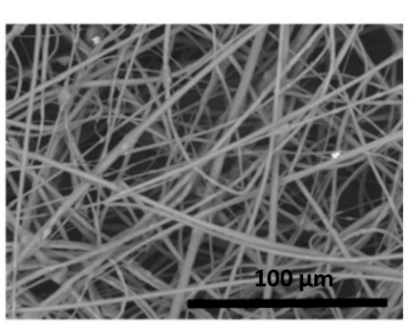

$\mathbf{F}$

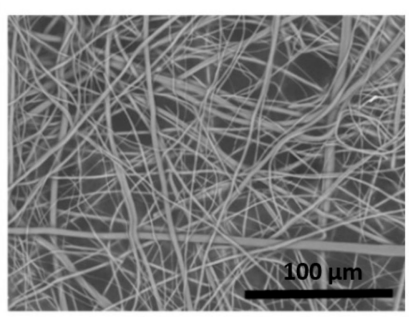

Figure 2. Quantification of collagen content by Sirius Red Assay in (A). PHBV films (ug of collagen per area) and 3D fibrous mesh fabricated by forcespinning (ug of collagen per mass of fibres) (B). Microscopy of 3D fibrous PHBV mesh stained with Sirus Red (C) before, and (D) after, collagen functionalisation. SEM image of fabricated scaffolds by forcespinning (E) before, and (F) after collagen grafting. Scale bar in SEM corresponds to $100 \mu \mathrm{m}$. 


\subsubsection{ATR-FTIR}

The results from the ATR-FTIR analysis of unconjugated PHBV fibre membranes and collagen-conjugated samples are shown in Figure 3. The strong band present at $1720 \mathrm{~cm}^{-1}$ corresponds to the $\mathrm{C}=\mathrm{O}$ group in the PHBV ester bond. The broad-band at $3100-3600 \mathrm{~cm}^{-1}$ in the conjugated samples is related to the $-\mathrm{OH}$ groups present in the collagen. Characteristic collagen amide bonds were observed at $1658 \mathrm{~cm}^{-1}$ and $1558 \mathrm{~cm}^{-1}$, representing Amide I and Amide II for the PHBV-Collagen-conjugated sample, respectively.

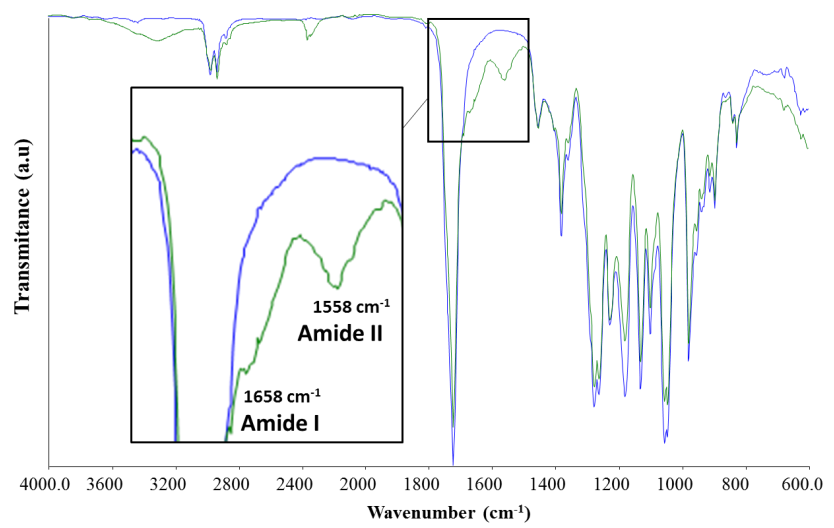

Figure 3. ATR-FTIR analysis of unconjugated (blue) and conjugated (green) fibrous scaffolds.

\subsubsection{XPS}

The surface chemical composition of the scaffolds was measured by XPS (Figure 4). PHBV presents two characteristic peaks of $\mathrm{C} 1 \mathrm{~s}, 284.5 \mathrm{eV}$, and $\mathrm{O} 1 \mathrm{~s}, 532.0 \mathrm{eV}$. With the conjugation of collagen, there is a sharp increase in the $\mathrm{N} 1 s$ signal at $398.7 \mathrm{eV}$, indicating that the conjugation was successful. Relative element content changed from $\mathrm{C} 1 s 74.5 \%$ (SD $\pm 1.8 \%$ ) to $70.5 \%$ (SD $\pm 0.8 \%$ ), $\mathrm{N} 1 s 1.7 \%$ (SD $\pm 0.6 \%$ ) to $10.3 \%$ (SD $\pm 0.7 \%$ ), and $\mathrm{O} 1 s 23.9 \%$ (SD $\pm 1.3 \%$ ) to $19.2 \%(\mathrm{SD} \pm 0.3 \%$ ), between $\mathrm{PHBV}$ and collagen-conjugated PHBV samples. High-resolution XPS analysis of the $\mathrm{N} 1 \mathrm{~s}$ signal detected the presence of $\mathrm{C}-\mathrm{N}(398.3 \mathrm{eV}),-\mathrm{NH}-$, and $\mathrm{C}-\mathrm{NH}_{2}(\sim 400 \mathrm{eV})$, and $\mathrm{C}-\mathrm{N}(401.5 \mathrm{eV})$ bonds in the conjugated samples.

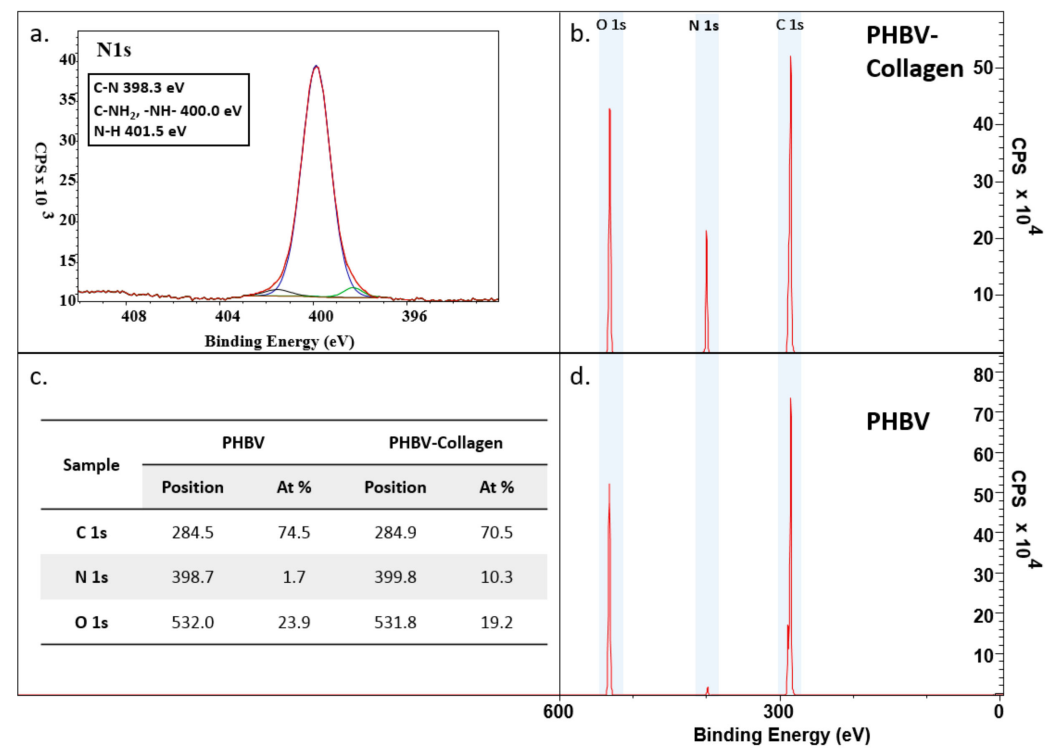

Figure 4. XPS analysis of PHBV and PHBV-Collagen fibre membranes: (a) a high-resolution view of the N 1s peak observed upon conjugation of collagen; (b) spectra for PHBV-Collagen; (c) table of peak binding energies and the percentage of elements present; and (d) spectra for PHBV. 


\subsubsection{Differential Scanning Calorimetry}

Figure 5 shows the DSC thermograms of both the conjugated and the unconjugated scaffolds, and the numerical data are summarised in the Table 1. Both thermograms displayed the characteristic twin peak of PHBV polymer melting behaviour, with a $T_{\mathrm{m}}$ (melting temperature) profile of $\mathrm{PHB}$ at $163.8^{\circ} \mathrm{C}$ and PHV (polyhydroxyvalerate) at $141.3^{\circ} \mathrm{C}$. However, for the PHBV-Collagen fibres, a decrease of the typical PHBV melting temperature for both PHB and PHV melting peaks upon collagen conjugation was noticed, with a $T_{\mathrm{m}}$ of $155.3^{\circ} \mathrm{C}$ for $\mathrm{PHB}$ and $136.9^{\circ} \mathrm{C}$ for PHV. No great differences were detected for $\Delta H_{\mathrm{m}}$ (melting enthalpy) between PHBV and PHBV-collagen-melting enthalpies. Crystallisation temperature, as $T_{\mathrm{c}}$ peaks, were recorded at 55.7 and $56.9 .6^{\circ} \mathrm{C}$ in unconjugated (PHBV) and collagen-conjugated (PHBV-Collagen) samples, respectively. By introducing collagen as a surface bio-functionalisation, $\Delta H_{\mathrm{c}}$ (crystallisation enthalpy) slightly decreased from 0.182 to $0.120 \mu \mathrm{V} \mathrm{s} / \mathrm{mg}$. Collagen denaturation temperature was detected as a small peak at $44.8^{\circ} \mathrm{C}$ for collagen-conjugated PHBV; this peak was not observed for pure PHBV scaffolds.

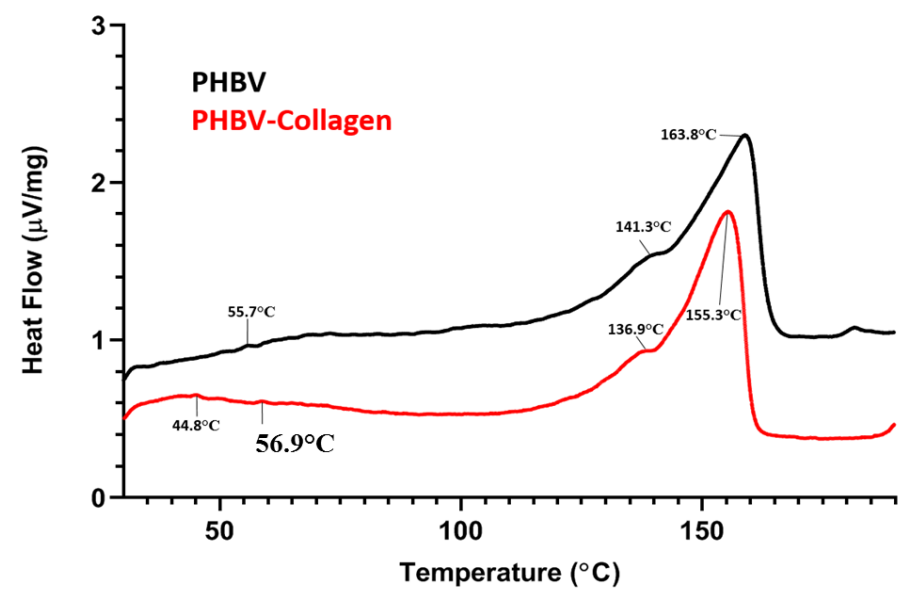

Figure 5. DSC thermograms of PHBV and PHBV-Collagen membranes.

Table 1. Summary of main peaks and the respective enthalpies of DSC thermal transitions for PHBV and PHBV-Collagen.

\begin{tabular}{|c|c|c|c|c|c|c|c|}
\hline Sample & $\begin{array}{c}\text { Collagen } \\
T_{\mathrm{d}}\left({ }^{\circ} \mathrm{C}\right)\end{array}$ & $\begin{array}{c}\text { PHBV } T_{\mathrm{c}} \\
\left({ }^{\circ} \mathrm{C}\right)\end{array}$ & $\begin{array}{c}\text { PHV } T_{\mathrm{m}} \\
\left({ }^{\circ} \mathrm{C}\right)\end{array}$ & $\begin{array}{l}\text { PHB } T_{\mathrm{m}} \\
\left({ }^{\circ} \mathrm{C}\right)\end{array}$ & $\begin{array}{c}\text { Collagen } \Delta H_{\mathrm{d}} \\
\quad(\mu \mathrm{V} \mathrm{s} / \mathrm{mg})\end{array}$ & $\begin{array}{c}\text { PHBV } \Delta H_{\mathrm{c}} \\
(\mu \mathrm{V} s / \mathrm{mg})\end{array}$ & $\begin{array}{c}\text { PHBV } \Delta H_{\mathrm{m}} \\
(\mu \mathrm{V} / \mathrm{mg})\end{array}$ \\
\hline PHBV & - & 55.7 & 141.3 & 163.8 & - & 0.182 & 150.4 \\
\hline PHBV-Collagen & 44.8 & 56.9 & 136.9 & 155.3 & 0.189 & 0.120 & 150.7 \\
\hline
\end{tabular}

\subsubsection{Contact Angle}

The difference in wettability between conjugated and unconjugated fibres is shown in Figure 6A, evaluated by static contact angle analysis. The contact angle of PHBV fibres was significantly $(p<0.001)$ reduced from an initial value of $92.56^{\circ}\left(\mathrm{SD} \pm 7.1^{\circ}\right)$ to $54.1^{\circ}\left(\mathrm{SD} \pm 9.5^{\circ}\right)$ upon conjugation with collagen.

\subsubsection{In Vitro Degradation Study}

The change in the mass of the samples incubated in phosphate buffered saline (PBS) (pH 7.4) was monitored over a six-week period (intervals T0, T1, T2, T4, and T6 weeks). Analysis of the dry start and finish weights (Figure 6B) indicated that there was no statistically significant difference in the mass between time points ( $\left.{ }^{*} \mathrm{~ns}\right)$ for both conjugated and unconjugated samples. However, by comparing both samples at the same time point (T), it was noticed that PHBV samples only showed a significant decrease in the mass against the PHBV-Collagen sample $\left({ }^{*} p<0.05\right)$ at week 6 (T6). 

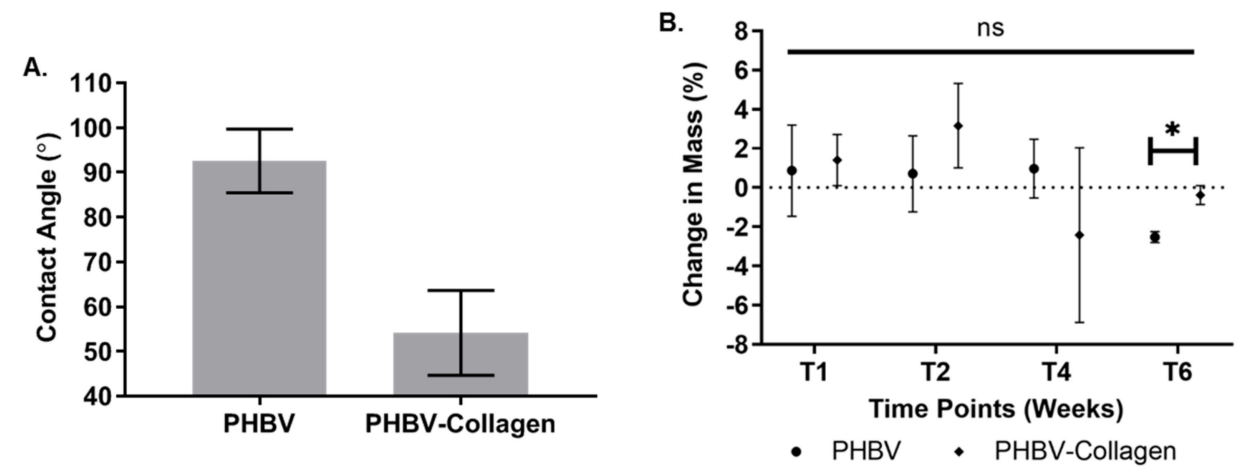

Figure 6. (A) Change in PHBV contact angle with collagen conjugation. (B) Percentage change in the mass in the conjugated and unconjugated fibres over six weeks of incubation at physiological conditions $\left({ }^{*} p<0.05\right.$, ns: not significant).

\subsection{Cytocompatibility Studies}

\subsubsection{Metabolic Activity}

The relative metabolic activity of Neo-NHDF fibroblasts was measured using a Presto Blue assay (Figure 7). At day 1, no significant difference in metabolic activity was observed between samples. On days 3 and 7, cells seeded onto PHBV-Collagen scaffolds had significantly higher metabolic output than those on PHBV scaffolds. On day 3 PHBV-Collagen samples displayed significantly higher cell metabolic activity than in the glass samples, while on day 7 , the difference in output was not significant.

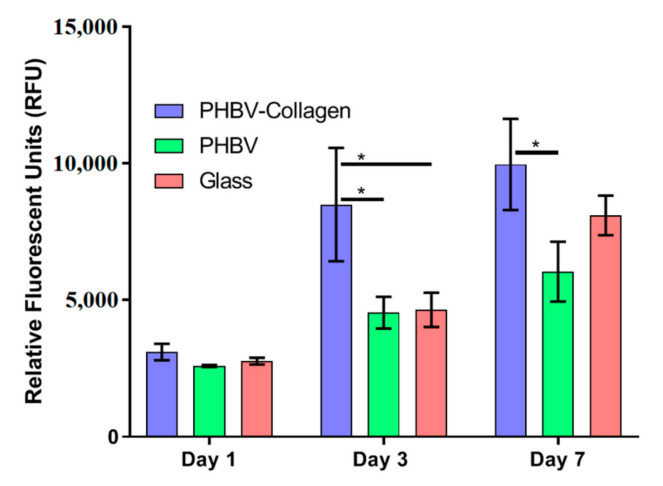

Figure 7. Relative fluorescent output of metabolised presto blue, representative of the cellular metabolic activity on scaffolds $\left({ }^{*} p<0.05\right)$.

\subsubsection{Cytocompatibility and Cell Morphology}

A Live/Dead assay was used to assess the viability of Neo-NHDF cells on the conjugated and unconjugated scaffolds, shown in Figure 8. On day 1, it can be observed that the number of dead cells on the PHBV-Collagen scaffolds was fewer than on the PHBV scaffolds. Simultaneously the PHBV-Collagen scaffolds were observed to host a much higher number of live cells than on the PHBV scaffolds.

In order to assess the cell morphology and cell spreading onto the samples, actin cytoskeleton staining of the cells was performed using rhodamine phalloidin. Figure 8 depicts the cell morphology of Neo-NHDF cells on conjugated and unconjugated scaffolds at days 1,3, and 7. The F-actin cytoskeleton staining evidenced a typical fibroblast morphology, with an increase over time when in the presence of the collagen conjugation (Figure 8). At different time points, cells were found to be more spread and widely attached to PHBV-Collagen scaffolds than on PHBV. In PHBV substrates, cell shape was rounded on day 1 (Figure 8), and more elongated towards day 7. Cells seeded onto PHBV-Collagen scaffolds were observed to attach and grow at a faster rate than on the PHBV scaffolds. 


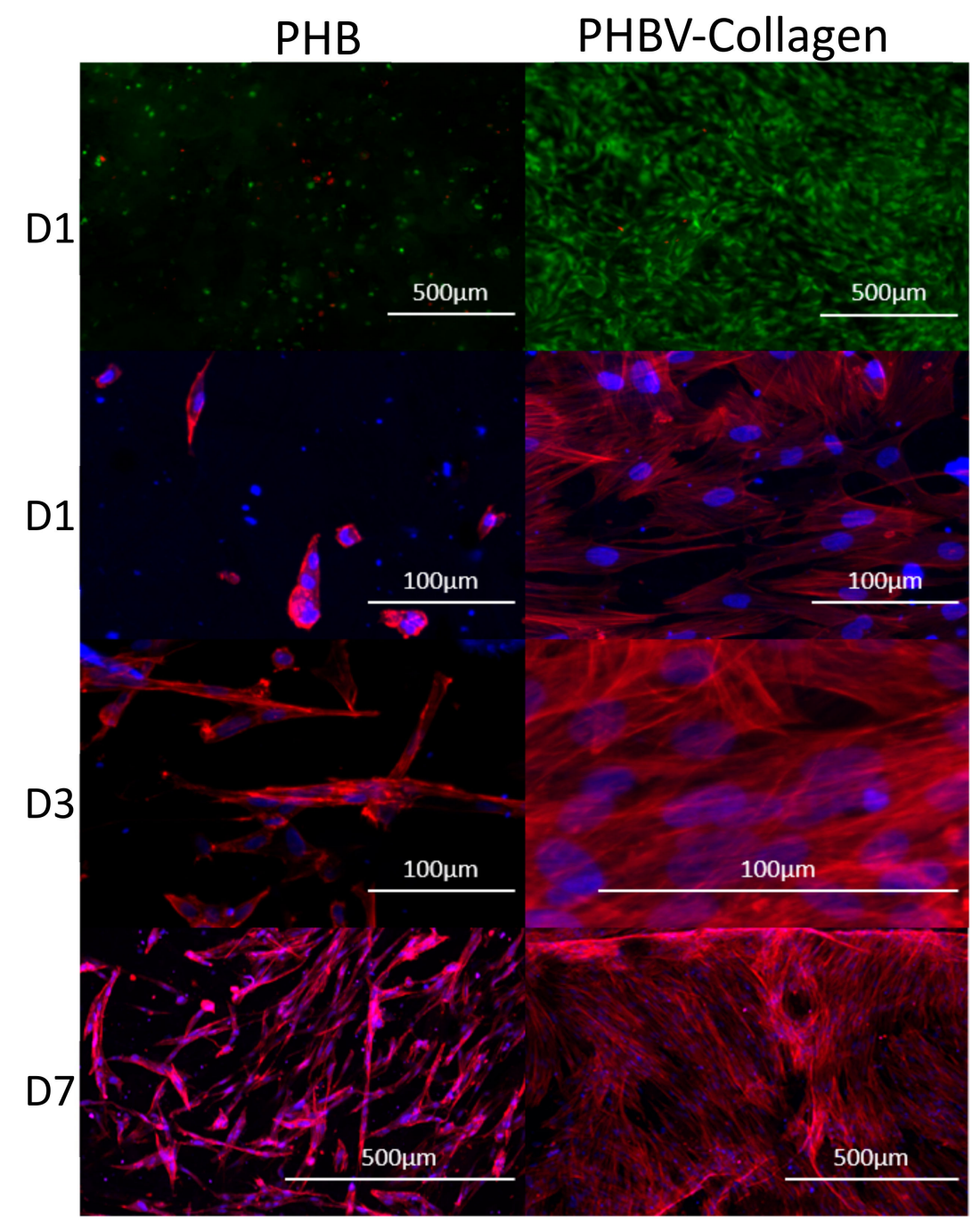

Figure 8. Cytotoxicity, cell viability, and cell morphology of Neo-NHDF cells cultured on force-spun PHBV scaffolds with and without collagen. Immunofluorescent images of Neo-NHDF cells stained with the Live/Dead membrane integrity assay (green-viable cells, red-dead cells, $5 \times$ magnification). Confocal microscope images showing the morphology of the Neo-NHDF cells seeded on PHBV and PHBV-Collagen scaffolds on days 1 (D1), 3 (D3), and 7 (D7) (red-phalloidin, and blue-DAPI, 5× and $10 \times$ magnifications).

\section{Discussion}

As cellular microenvironments play a relevant role in cellular function, mimicking the physical and chemical properties of native extracellular matrices is a useful approach to regulate cell behaviour. A key factor in tissue engineering and regenerative medicine is the design and manufacture of biocompatible and biodegradable constructs. PHBV is a promising biodegradable and non-antigenic biopolymer, as low molecular weight PHB is present in biological cells. Although this biopolymer (and monomers and oligomers) has been shown to be an excellent candidate biomaterial for tissue engineering [29], similar work on nerve and skin tissue engineering have shown improved surface properties and cellular behaviour on collagen-coated nanofibers $[4,5,18]$. Collagen is a key component of the extracellular matrix present in nearly every tissue in the human body; thus it's grafting to a base polymer has been shown to increase the adhesion and proliferation of cells onto polymeric scaffolds [30], making collagen functionalisation treatment an attractive practice for enhancing the biological performance of a material. 
In this work, the grafting of collagen to PHBV forcespun membranes was confirmed by XPS and ATR-FTIR as successful. Characteristic FTIR collagen signals corresponding to Amide I and Amide II [31] were identified in the conjugated samples. This result was reinforced by XPS analysis, where an appreciable increase in the $\mathrm{N} 1 \mathrm{~s}$ signal intensity was observed in conjugated samples. Nitrogen from the collagen-conjugated PHBV constituted about 10.3 at \% of the total atom. Although an N 1 s signal was detected in the low-resolution XPS scan of unconjugated PHBV samples, the signal intensity of 1.7 at \% was within the analytical error, as the structure of PHBV did not contain nitrogen. Deconvoluted high-resolution XPS of the $\mathrm{N} 1$ s peak in the conjugated samples revealed the presence of $\mathrm{C}-\mathrm{N},-\mathrm{NH}-$, $\mathrm{C}-\mathrm{NH}_{2}$, and $\mathrm{N}-\mathrm{H}$ attributable to signals between 398.3 and $401.5 \mathrm{eV}$. The bond signals closely matched the typical values for collagen, corresponding with both the literature [32-34] and the Thermo Scientific XPS library.

Moreover, the Sirius Red staining assay was used to qualitatively and quantitatively assess the presence of structural collagen in the fibre scaffolds. The dye functions by binding to the side chains present only in the structural collagen; gelatin, or otherwise denatured collagen is not bound by the dye [35]. Images of the scaffolds after staining show a high level of collagen conjugation, a result confirmed by the quantitative measurement of collagen content. Fibre membranes were observed to contain a much higher level of collagen content by mass than films of PHBV. The higher levels of collagen present in the fibres can be attributed to the higher surface area [36] exhibited by nano/microfibers in comparison to films, exposing more sites of conjugation in the fibre membranes.

Analysis of the surface wettability demonstrated an increase in the hydrophilicity of PHBV fibres upon conjugation due to the hydrophilic structural motifs present in the collagen molecule. A significant reduction from $93^{\circ}$ to $54^{\circ}$ in the contact angle of deionised water with conjugation is indicative of more favourable cellular adhesion properties [37,38]. A general trend is an increase in cellular adhesion on surfaces with a lower wettability value; however, this property consists of multiple factors, such as surface roughness and surface chemistry [39], which independently affect wettability and cellular adhesion. PHBV is quite a hydrophobic polyester without many functional groups for biomolecule grafting. Therefore, these results indicated that the chemical grafting of collagen motifs [40] increases the wettability of PHBV, which enhances cellular adhesion as observed in this study.

The DSC analysis was used to assess the thermal properties of PHBV and PHBV grafted collagen. Both spectra exhibited typical $T_{\mathrm{c}}$ and $T_{\mathrm{m}}$ values for PHBV [41], with the presence of the characteristic peak for collagen's denaturation temperature only in the conjugated samples [42]. Collagen conjugation led to a small downward shift in peak temperatures for the melting points of PHB and PHV, with peak enthalpies remaining consistent. Surface functionalisation or blending of proteins is known to decrease the melting temperature of polymeric-based scaffolds, and this study shows that the addition of collagen reduced the melting temperature of PHBV $[18,43]$. However, the $T_{\mathrm{C}}$ of PHBV membranes remained similar, showing small changes in the total crystallinity. These results indicate that collagen was successfully grafted superficially onto the PHBV forcespun fibres, without decreasing or impacting the polymer bulk thermal properties. The thermal stability of the collagen conjugated substrates remains comparable to neat PHBV.

Similarly, analysis of the data collected from in vitro degradation studies showed no significant change in mass over the course of six weeks, in both sample sets. This result is consistent with the observation of similar thermal properties in conjugated and unconjugated samples, in that surface conjugation with collagen presents no significant change to the stability of fibres under degradation conditions; a result consistent with other studies on PHBV fibre scaffolds [18]. These results are consistent with the measured degradation of PHBV in the literature, which exhibits a slow degradation rate under identical conditions, of 5.6\% total mass loss after 229 days [44]. In addition, these mass changes are attributed solely to the PHBV, as the mass of collagen would be too low to impact the results. The retention of collagen in its triple helical structure under degradation conditions is a 
good indicator that the conjugated fibres can retain their enhanced biological characteristics over time in vivo.

A key component in determining the viability of the scaffold is to observe the behaviour of the fibroblastic cells on the membranes. Collagen is a major constituent of the ECM, bearing the motifs to which cell integrins bind [45], and as such, scaffolds conjugated with collagen displayed a much higher level of cellular growth and adhesion [20,21,46]. A Live/Dead assay (Figure 8) on the same sample area showed a greater quantity of live cells on PHBV-Collagen than the PHBV surface. Cellular viability is a key indicator of biological success in living tissue, and thus an important aspect of assessing the efficacy of new materials for TE applications [47]. In this work, a greater number of viable cells growing on PHBV-Collagen conjugated substrates, with an increase in metabolic activity after day 3 was found (as shown in Figure 7). As the adhesion of cells to a surface is key in the mediation of multiple cell functions [38,48] (i.e., structural protein deposition, proliferation, migration, etc.), greater observed viability, mitochondrial activity, cell attachment, and spreading was observed in the presence of collagen motif-bearing conjugated membranes. Although not in collagen-grafted PHBV membranes, similar results were observed by the studies of Prabhakaran et al. using PC12 neural cells on electrospun PHBV nanofibers, where cell proliferation improved on the blended PHBV-Collagen nanofibers for nerve tissue engineering [18].

Confocal imaging of the fibroblast cytoskeleton (F-actin) and nuclei evidenced normal fibroblast morphology with an increased number of cells over time, specifically in the presence of collagen. By day 7, cells formed a dense monolayer on the surface of conjugated scaffolds, orienting themselves nearly parallel to each other and the substrate. Whereas cells growing on the unconjugated scaffolds were more sparsely distributed. Human dermal fibroblasts attachment, growth, spreading, and viability were significantly enhanced on the PHBV-Collagen, demonstrating the positive effect of collagen conjugation to promote tissue growth and overcome relatively poor cell adhesion properties of the PHBV material. Similar work for cartilage tissue engineering showed that collagen-modified PHBV provided a more favourable surface for chondrocytes adhesion, spread, and proliferation than PHBV [25]. With the conjugation of collagen, there are a greater number of focal adhesion points available for cells to adhere to, allowing the cells to begin proliferation sooner than without focal adhesion points. This results in quicker tissue formation of tissue, ideal for TE scaffolds, where rapid regeneration of the host tissue is a primary goal.

\section{Conclusions}

PHBV fibre scaffolds were successfully prepared by force-spinning and covalently conjugated with type I collagen by using carbodiimide chemistry. Collagen surface functionalisation was evidenced by the presence of amide I and II bands in ATR-FTIR and nitrogen contents detected by XPS scans of about 10.3 at $\%$ of the total atom, which was not observed in pure PHBV scaffolds. Surface functionalisation improved the surface properties of PHBV substrates significantly by rendering it hydrophilic properties, evidenced by a significant reduction of PHBV contact angles from $93^{\circ}$ to $54^{\circ}$ by grafting collagen. The amount of collagen was successfully quantified in (2D) films by obtaining a superficial amount of $8 \mu \mathrm{g} / \mathrm{cm}^{-2}$ collagen and in 3D membranes with $25 \mu \mathrm{g}$ of collagen per mg of porous membranes. Collagen grafting onto PHBV forcespun membranes did not change the morphology significantly and the fibre diameters, ranging between $631 \mathrm{~nm}$ and $6.85 \mu \mathrm{m}$. Moreover, collagen-conjugated PHBV fibres showed physiological stability as pure PHBV scaffolds, as degradation assays showed no changes of mass over six weeks of incubation and the thermal properties of PHBV polymer were maintained post chemical conjugation. Collagen-modified PHBV forcespun membranes provided a more favourable surface for human fibroblast adhesion, spread, and growth than PHBV. Force-spun PHBV fibre membranes conjugated with type I collagen presenting promising characteristics for use as scaffolds for tissue engineering, as an alternative and scalable method of creating nano/microfibres with enhanced biological properties in a more efficient and less toxic manner than current technologies. 
Author Contributions: Conceptualization, A.M.F., S.R., K.D. and P.G.; Methodology, K.I.S.M.-B., M.T. and S.U.; Formal Analysis, K.I.S.M.-B., P.G., K.D., and A.M.F.; Investigation, K.I.S.M.-B., S.U., S.R.; Data Curation, K.I.S.M.-B., P.G., K.D., and A.M.F.; Writing-Original Draft Preparation, K.I.S.M.-B., S.U. and A.M.F.; Writing-Review \& Editing, K.I.S.M.-B., S.U., A.M.F., K.D. and P.G.; Supervision, A.M.F., K.D. and P.G.

Acknowledgments: The authors would like to thank the EPSRC Centre for Doctoral Training in Additive Manufacturing and 3D Printing (EP/L01534X/1), the EPSRC Centre for Innovative Manufacture in Medical Devices (EP/K029592/1), and the Tissue Engineering and Regenerative Therapies Centre Versus Arthritis (Award 21156). Data supporting this publication is openly available under an 'Open Data Commons Open Database License'. Additional metadata are available at: http://dx.doi.org/10.25405/data.ncl.8189126. Please contact Newcastle Research Data Service at rdm@ncl.ac.uk for access instructions.

Conflicts of Interest: The authors declare no conflict of interest.

\section{References}

1. Williams, S.F.; Martin, D.P.; Horowitz, D.M.; Peoples, O.P. PHA applications: Addressing the price performance issue I. Tissue engineering. Int. J. Biol. Macromol. 1999, 25, 111-121. [CrossRef]

2. Han, I.; Shim, K.J.; Kim, J.Y.; Im, S.U.; Sung, Y.K.; Kim, M.; Kang, I.-K.; Kim, J.C. Effect of poly(3hydroxybutyrate-co-3-hydroxyvalerate) nanofiber matrices cocultured with hair follicular epithelial and dermal cells for biological wound dressing. Artif. Organs 2007, 31, 801-808. [CrossRef]

3. Chen, G.; Wu, Q. The application of polyhydroxyalkanoates as tissue engineering materials. Biomaterials 2005, 26, 6565-6578. [CrossRef]

4. Ghebi, A.; Khoshnevisan, K.; Ketabchi, N.; Derakhshan, M.A.; Babadi, A.A. Application of electrospun nanofibrous PHBV scaffold in neural graft and regeneration: A mini-review. Nanomed. Res. J. 2016, 1, 107-111.

5. Keshel, S.H.; Biazar, E.; Rezaei, T.M.; Rahmati, R.M.; Ronaghi, A.; Ebrahimi, M.; Rad, H.; Sahebalzamani, A.; Rakhshan, A.; Afsordeh, K. The healing effect of unrestricted somatic stem cells loaded in collagen-modified nanofibrous PHBV scaffold on full-thickness skin defects. Artif. Cells Nanomed. Biotechnol. 2004, 42, $210-216$. [CrossRef]

6. Vasita, R.; Katti, D.S. Nanofibers and their applications in tissue engineering. Int. J. Nanomed. 2006, 1, 15-30. [CrossRef]

7. Murphy, C.M.; Haugh, M.G.; O'Brien, F.J. The effect of mean pore size on cell attachment, proliferation and migration in collagen-glycosaminoglycan scaffolds for bone tissue engineering. Biomaterials 2010, 31, 461-466. [CrossRef]

8. Sarkar, K.; Gomez, C.; Zambrano, S.; Ramirez, M.; de Hoyos, E.; Vasquez, H.; Lozano, K. Electrospinning to forcespinning $^{\mathrm{TM}}$. Mater. Today 2010, 13, 12-14. [CrossRef]

9. Upson, S.J.; O’Haire, T.; Russell, S.J.; Dalgarno, K.; Ferreira, A.M. Centrifugally spun PHBV micro and nanofibres. Mater. Sci. Eng. C 2017, 76, 190-195. [CrossRef]

10. Veleirinho, B.; Coelho, D.S.; Dias, P.F.; Maraschin, M.; Ribeiro-do-Valle, R.M.; Lopes-da-Silva, J.A. Nanofibrous poly(3-hydroxybutyrate-co-3-hydroxyvalerate)/chitosan scaffolds for skin regeneration. Int. J. Biol. Macromol. 2012, 51, 343-350. [CrossRef]

11. Veleirinho, B.; Ribeiro-do-Valle, R.M.; Lopes-da-Silva, J.A. Processing conditions and characterization of novel electrospun poly(3-hydroxybutyrate-co-hydroxyvalerate)/chitosan blend fibers. Mater. Lett. 2011, 65, 2216-2219. [CrossRef]

12. Peschel, G.; Dahse, H.-M.; Konrad, A.; Wieland, G.D.; Mueller, P.-J.; Martin, D.P.; Roth, M. Growth of keratinocytes on porous films of poly(3-hydroxybutyrate) and poly(4-hydroxybutyrate) blended with hyaluronic acid and chitosan. J. Biomed. Mater. Res. Part A 2008, 85, 1072-1081. [CrossRef]

13. Wang, X.; Yan, H.; Zhou, Y.; Lou, X.; Zhang, Y. Fabrication of fibrous PLLA/PHBV scaffolds with shape memory capability. J. Control. Release 2017, 259, e144-e145. [CrossRef]

14. Thadavirul, N.; Pavasant, P.; Supaphol, P. Fabrication and evaluation of polycaprolactonepoly(hydroxybutyrate) or poly(3-hydroxybutyrate-co-3-hydroxyvalerate) dual-leached porous scaffolds for bone tissue engineering applications. Macromol. Mater. Eng. 2017, 302, 1600289. [CrossRef]

15. Li, W.; Nooeaid, P.; Roether, J.A.; Schubert, D.W.; Boccaccini, A.R. Preparation and characterization of vancomycin releasing PHBV coated $45 \mathrm{~S} 5$ Bioglass ${ }^{\circledR}$-based glass-ceramic scaffolds for bone tissue engineering. J. Eur. Ceram. Soc. 2014, 34, 505-514. [CrossRef] 
16. Li, W.; Ding, Y.; Rai, R.; Roether, J.A.; Schubert, D.W.; Boccaccini, A.R. Preparation and characterization of PHBV microsphere/45S5 bioactive glass composite scaffolds with vancomycin releasing function. Mater. Sci. Eng. C 2014, 41, 320-328. [CrossRef]

17. Sajesh, K.M.; Kiran, K.; Nair, S.V.; Jayakumar, R. Sequential layer-by layer electrospinning of nano $\mathrm{SrCO}_{3} / \mathrm{PRP}$ loaded PHBV fibrous scaffold for bone tissue engineering. Compos. Part B Eng. 2016, 99, 445-452. [CrossRef]

18. Prabhakaran, M.P.; Vatankhah, E.; Ramakrishna, S. Electrospun aligned PHBV/collagen nanofibers as substrates for nerve tissue engineering. Biotechnol. Bioeng. 2013, 110, 2775-2784. [CrossRef]

19. Gelse, K.; Pöschl, E.; Aigner, T. Collagens-Structure, function, and biosynthesis. Adv. Drug Deliv. Rev. 2003, 55, 1531-1546. [CrossRef]

20. Kiran, S.; Nune, K.C.; Misra, R.D.K. The significance of grafting collagen on polycaprolactone composite scaffolds: Processing-structure-functional property relationship. J. Biomed. Mater. Res. Part A 2015, 103, 2919-2931. [CrossRef]

21. Sousa, I.; Mendes, A.; Bártolo, P.J. PCL scaffolds with collagen bioactivator for applications in tissue engineering. Procedia Eng. 2013, 59, 279-284. [CrossRef]

22. Cui, M.; Liu, L.; Guo, N.; Su, R.; Ma, F. Preparation, cell compatibility and degradability of collagen-modified poly(lactic acid). Molecules 2015, 20, 595-607. [CrossRef]

23. Castillo-Briceño, P.; Bihan, D.; Nilges, M.; Hamaia, S.; Meseguer, J.; García-Ayala, A.; Farndale, R.W.; Mulero, V. A role for specific collagen motifs during wound healing and inflammatory response of fibroblasts in the teleost fish gilthead seabream. Mol. Immunol. 2011, 48, 826-834. [CrossRef]

24. Ferreira, A.M.; Gentile, P.; Chiono, V.; Ciardelli, G.A. Collagen for bone tissue regeneration. Acta Biomater. 2012, 8, 3191-3200. [CrossRef]

25. Wang, Y.; Ke, Y.; Ren, L.; Wu, G.; Chen, X.; Zhao, Q. Surface engineering of PHBV by covalent collagen immobilization to improve cell compatibility. J. Biomed. Mater. Part A 2009, 88, 616-627. [CrossRef]

26. Ai, J.; Keshel, S.H.; Ghorbani, F.; Ejazi, F.; Biazar, E.; Asefnejad, A.; Pourshamsian, K.; Montazeri, M. Fabrication of coated-collagen electrospun PHBV nanofiber film by plasma method and its cellular study. J. Nanomater. 2011, 2011, 123724. [CrossRef]

27. Zeugolis, D.I.; Khew, S.T.; Yew, E.S.Y.; Ekaputra, A.K.; Tong, Y.W.; Yung, L.-Y.L.; Hutmacher, D.W.; Sheppard, C.; Raghunath, M. Electro-spinning of pure collagen nano-fibres-Just an expensive way to make gelatin? Biomaterials 2008, 29, 2293-2305. [CrossRef]

28. Hexafluoroisopropanol MSDS, Sigma Aldrich. Available online: http://www.sigma-aldrich.com (accessed on 3 May 2019).

29. Lü, L.-X.; Wang, Y.-Y.; Mao, X.; Xiao, Z.-D.; Huang, N.-P. The effects of PHBV electrospun fibers with different diameters and orientations on growth behavior of bone-marrow-derived mesenchymal stem cells. Biomed. Mater. 2012, 7, 015002. [CrossRef]

30. Gentile, P.; McColgan-Bannon, K.; Gianone, N.C.; Sefat, F.; Dalgarno, K.; Ferreira, A.M. Biosynthetic PCL-graft-collagen bulk material for tissue engineering applications. Materials 2017, 10, 693. [CrossRef]

31. De Campos Vidal, B.; Mello, M.L.S. Collagen type I amide I band infrared spectroscopy. Micron 2011, 42, 283-289. [CrossRef]

32. Zhang, X.-Y.; Zheng, Y.; Liu, C.-H.; Wang, P.-H.; Zhu, Y.-Y. Facile and large scale in situ synthesis of the thermal responsive fluorescent SiNPs/PNIPAM hydrogels. RSC Adv. 2016, 6, 55666-55670. [CrossRef]

33. Dementjev, A.; de Graaf, A.; van de Sanden, M.C.M.; Maslakov, K.I.; Naumkin, A.V.; Serov, A.A. X-ray photoelectron spectroscopy reference data for identification of the $\mathrm{C}_{3} \mathrm{~N}_{4}$ phase in carbon-nitrogen films. Diam. Relat. Mater. 2000, 9, 1904-1907. [CrossRef]

34. Diller, K.; Klappenberger, F.; Allegretti, F.; Papageorgiou, A.C.; Fischer, S.; Duncan, D.A.; Maurer, R.J.; Lloyd, J.A.; Oh, S.C.; Reuter, K. Temperature-dependent templated growth of porphine thin films on the (111) facets of copper and silver. J. Chem. Phys. 2014, 141, 144703. [CrossRef] [PubMed]

35. Dapson, R.W.; Fagan, C.; Kiernan, J.A.; Wickersham, T.W. Certification procedures for sirius red F3B (CI 35780, Direct red 80). Biotech. Histochem. 2011, 86, 133-139. [CrossRef] [PubMed]

36. Ramakrishna, S.; Fujihara, K.; Teo, W.-E.; Yong, T.; Ma, Z.; Ramaseshan, R. Electrospun nanofibers: Solving global issues. Mater. Today 2006, 9, 40-50. [CrossRef]

37. Keshel, S.H.; Azhdadi, S.N.K.; Asefnezhad, A.; Sadraeian, M.; Montazeri, M.; Biazar, E. The relationship between cellular adhesion and surface roughness for polyurethane modified by microwave plasma radiation. Int. J. Nanomed. 2011, 6, 641-647. 
38. Khalili, A.A.; Ahmad, M.R. A review of cell adhesion studies for biomedical and biological applications. Int. J. Mol. Sci. 2015, 16, 18149-18184. [CrossRef]

39. Dowling, D.P.; Miller, I.S.; Ardhaoui, M.; Gallagher, W.M. Effect of surface wettability and topography on the adhesion of osteosarcoma cells on plasma-modified polystyrene. J. Biomater. Appl. 2011, 26, 327-347. [CrossRef]

40. Yuan, S.; Xiong, G.; Wang, X.; Zhang, S.; Choong, C. Surface modification of polycaprolactone substrates using collagen-conjugated poly(methacrylic acid) brushes for the regulation of cell proliferation and endothelialisation. J. Mater. Chem. 2012, 22, 13039-13049. [CrossRef]

41. Shang, L.; Fei, Q.; Zhang, Y.H.; Wang, X.Z.; Fan, D.-D.; Chang, H.N. Thermal properties and biodegradability studies of poly(3-hydroxybutyrate-co-3-hydroxyvalerate). J. Polym. 2011, 20, 23-28. [CrossRef]

42. Leikina, E.; Mertts, M.V.; Kuznetsova, N.; Leikin, S. Type I collagen is thermally unstable at body temperature. Proc. Natl. Sci. Acad. USA 2002, 99, 1314-1318. [CrossRef] [PubMed]

43. Ke, Y.; Wang, Y.; Ren, L.; Wu, G.; Xue, W. Surface modification of PHBV films with different functional groups: Thermal properties and in vitro degradation. J. Appl. Polym. Sci. 2010, 118, 390-398. [CrossRef]

44. Amass, W.; Amass, A.; Tighe, B. A review of biodegradable polymers: uses, current developments in the synthesis and characterization of biodegradable polyesters, blends of biodegradable polymers and recent advances in biodegradation studies. Polym. Int. 1998, 47, 89-144. [CrossRef]

45. Heino, J. The collagen family members as cell adhesion proteins. BioEssays 2007, 29, 1001-1010. [CrossRef] [PubMed]

46. Sousa, I.; Mendes, A.; Pereira, R.F.; Bártolo, P.J. Collagen surface modified poly( $\varepsilon$-caprolactone) scaffolds with improved hydrophilicity and cell adhesion properties. Mater. Lett. 2014, 134, 263-267. [CrossRef]

47. Insomphun, C.; Chuah, J.-A.; Kobayashi, S.; Fujiki, T.; Numata, K. Influence of hydroxyl groups on the cell viability of polyhydroxyalkanoate (PHA) scaffolds for tissue engineering. ACS Biomater. Sci. Eng. 2017, 3, 3064-3075. [CrossRef]

48. Matschegewski, C.; Staehlke, S.; Loeffler, R.; Lange, R.; Chai, F.; Kern, D.P.; Beck, U.; Nebe, B.J. Cell architecture-cell function dependencies on titanium arrays with regular geometry. Biomaterials 2010, 31, 5729-5740. [CrossRef] 